\title{
Distributionally Robust Joint Chance Constrained Problem under Moment Uncertainty
}

\author{
Ke-wei Ding ${ }^{1,2}$ \\ ${ }^{1}$ Department of Foundation Education, Southwest University for Nationalities, Chengdu, Sichuan 610041, China \\ ${ }^{2}$ Department of Mathematics, Sichuan University, Chengdu, Sichuan 610064, China
}

Correspondence should be addressed to Ke-wei Ding; bluedkw@163.com

Received 7 April 2014; Accepted 30 May 2014; Published 15 June 2014

Academic Editor: Nan-Jing Huang

Copyright (c) 2014 Ke-wei Ding. This is an open access article distributed under the Creative Commons Attribution License, which permits unrestricted use, distribution, and reproduction in any medium, provided the original work is properly cited.

\begin{abstract}
We discuss and develop the convex approximation for robust joint chance constraints under uncertainty of first- and secondorder moments. Robust chance constraints are approximated by Worst-Case CVaR constraints which can be reformulated by a semidefinite programming. Then the chance constrained problem can be presented as semidefinite programming. We also find that the approximation for robust joint chance constraints has an equivalent individual quadratic approximation form.
\end{abstract}

\section{Introduction}

Chance constraints, also called probabilistic constraints in the literature, have a long history in stochastic programming and are the direct way to treat stochastic data uncertainty. With a large class problem involved, it can be formulated in the following form:

$$
\begin{array}{ll}
\min _{\mathbf{x} \in \mathbb{R}^{n}} & \mathbf{c}^{T} \mathbf{x} \\
\text { s.t. } & \mathbb{P}_{\boldsymbol{\xi}}\left(w_{i}^{0}(\mathbf{x})+\mathbf{w}_{i}(\mathbf{x})^{T} \boldsymbol{\xi} \leq 0, \forall i=1,2, \ldots, m\right) \geq 1-\epsilon, \\
& \\
& \mathbf{x} \in \chi,
\end{array}
$$

where $\boldsymbol{\xi}$ is undetermined vector, $\mathbf{x} \in \chi$ is the decision vector, $\chi \in \mathbb{R}^{n}$ is a bounded, convex closed set which can be represented by a set of additional deterministic semidefinite constraints, and $\mathbf{c} \in \mathbb{R}^{n}$ is the deterministic cost vector. The chance constraint in the above problem requires all the $m$ uncertainty-affected constraints to be jointly feasible with probability at least $1-\epsilon$, where $\epsilon$ is a desired safety factor given by the decision-maker. Problem (1) can be classified as an individual chance constrained problem when $m=1$ and a joint chance constrained problem when $m \geq 2$.

This problem has been considered by Charnes et al. [1], Miller and Wagnet [2], and Prékopa [3]. Due to the feasible set of problems, (1) is typically nonconvex and sometimes even disconnected; at the same time, the full and accurate information about the probability distribution $\mathbb{P}_{\xi}$ cannot be required; the problem has not found interest and wide application in theory and practice for a long time.

One interesting issue on the chance constrained problem is to determine the distributional condition under which the problem can be reformulated as tractable convex programming. Alizadeh and Goldfarb [4], Calafiore and EI Ghaoui [5], Erdoğan and Iyengar [8], and Zymler et al. [6] showed that the chance constraint can be reformulated as tractable convex and cone constraints under some special exact information, respectively. However, the computation of chance constrained problems for general case is intractable. Nemirovski and Shapiro [7] pointed out that computing the probability of a weighted sum of uniformly distributed variables being nonpositive is already NP-hard. The intractability of a chance constrained problem using exact information has spurred recent interest in robust optimization in which data uncertainties are controlled in several types of uncertainty sets $[9,10]$. Moreover, robust optimization generally needs segmental information on probability distributions such as known supports and covariances. Zymler et al. [6] showed an exact LMIS reformulation for chance constrained problem which can be computed by solving a tractable SDP under 
known first- and second-order moments information. Usually, in practice, one only has limited information about the probability distribution driving the uncertain parameters, involved in the decision-making process. It implies that we cannot obtain the exact moments information. In this paper, we extend the framework of Zymler et al. [6] to the case of inexact known moments information. We use the following two constraints parameterized by $\eta \geq 0$ which rely on empirical estimates of the mean $\boldsymbol{\mu}_{0}$ and covariance matrix $\Sigma_{0}$ of the random vector to construct the distributional information:

$$
\begin{gathered}
\boldsymbol{\mu}_{0}-\boldsymbol{\eta} \leq \mathbb{E}[\boldsymbol{\xi}] \leq \boldsymbol{\mu}_{0}+\boldsymbol{\eta}, \\
\boldsymbol{\Sigma}_{0}+\left(\boldsymbol{\mu}_{0}-\boldsymbol{\eta}\right)\left(\boldsymbol{\mu}_{0}-\boldsymbol{\eta}\right)^{T} \\
\preceq \mathbb{E}\left[\xi \xi^{T}\right] \preceq \boldsymbol{\Sigma}_{0}+\left(\boldsymbol{\mu}_{0}+\boldsymbol{\eta}\right)\left(\boldsymbol{\mu}_{0}+\boldsymbol{\eta}\right)^{T} .
\end{gathered}
$$

It describes how likely $\boldsymbol{\xi}$ is to be close to $\boldsymbol{\mu}_{0}$ controlled by the vector $\boldsymbol{\eta}$. At the same time, the parameter $\boldsymbol{\eta}$ provides natural means of quantifying one's confidence in $\boldsymbol{\mu}_{0}$ and $\boldsymbol{\Sigma}_{0}$. In what follows, we consider the latest problem in this paper under the distributional set

$$
\begin{aligned}
& \mathbb{D}\left(\mathbb{R}^{L}, \boldsymbol{\mu}_{0}, \boldsymbol{\Sigma}_{0}, \boldsymbol{\eta}\right) \\
& =\left\{\mathbb{P} \in \mathscr{P}: \mathbb{P}\left(\xi \in \mathbb{R}^{L}\right)=1 \text { and satisfies }(2)\right\},
\end{aligned}
$$

where $\mathbb{D}$ is the set of all probability distributions on the measurable space $\left(\mathbb{R}^{L}, \mathbb{B}\right)$ and $\mathbb{B}$ is the Borel $\sigma$-algebra on $\mathbb{R}^{L}$. To this end, let $\mathscr{P}$ denote the set of all probability measures corresponding to $\mathbb{D}$.

Now let us consider the following distributionally robust chance constrained program:

$$
\inf _{\mathbb{P} \in \mathscr{P}} \mathbb{P}\left(w_{i}^{0}(\mathbf{x})+\mathbf{w}_{i}(\mathbf{x})^{T} \boldsymbol{\xi} \leq 0, \forall i=1,2, \ldots, m\right) \geq 1-\epsilon
$$

It is easy to verify that the feasible set of the above inequality is a subset of the feasible set of problem (1). This yields the following distributionally robust chance constrained program:

$$
\begin{array}{ll}
\min _{\mathbf{x} \in \mathbb{R}^{n}} & c^{T} x \\
\text { s.t. } & \inf _{\mathbb{P} \in \mathscr{P}} \mathbb{P}\left(w_{i}^{0}(\mathbf{x})+\mathbf{w}_{i}(\mathbf{x})^{T} \xi \leq 0, \forall i=1,2, \ldots, m\right) \geq 1-\epsilon,
\end{array}
$$

which constitutes a conservative approximation for problem (1) in the sense that it has the same objective functions but a smaller feasible set.

In this paper, we discuss approximations for distributionally robust joint chance constraints under inexact information of first- and second-order moments, which extends the framework of Zymler et al. [6] from exact known first- and second-order moments. We prove that it can be approximated by a Worst-Case CVaR constraint which can be represented as a semidefinite programming. Then, we show that distributionally robust joint chance constrained problem has an equivalent quadratic approximation form. The advantage of the new framework lies on the limited information about distribution and the tractable convex approximation.

\section{Distributionally Robust Joint Chance Constraints for $L P$}

Let $g(\mathbf{x}, \boldsymbol{\xi})$ be the chance constraint with the decision vector $\mathbf{x}$ and the random vector $\boldsymbol{\xi}$. Now, we consider the general robust individual chance constraint

$$
\inf _{\mathbb{P} \in \mathscr{P}} \mathbb{P}(g(\mathbf{x}, \boldsymbol{\xi}) \leq 0) \geq 1-\epsilon,
$$

whose feasible set is denoted by $\Pi_{g}=\left\{x \in \mathbb{R}^{n}\right.$ : $\left.\inf _{\mathbb{P} \in \mathscr{P}} \mathbb{P}(g(x, \xi) \leq 0) \geq 1-\epsilon\right\}$. Shapiro et al. [11] showed that the feasible set is convex if the probability distribution function is $\alpha$-concave and $g(\mathbf{x}, \boldsymbol{\xi})$ are quasiconcave jointly in both arguments. Unfortunately, the above chance constraint is not necessarily convex in the decision variables $\mathbf{x}$.

It is well-known that CVaR method, popularized by Rockafellar and Uryasev [12], is the tightest convex approximation to the general individual probabilistic constraint (see, e.g., $[7,13])$. Then, using the CVaR method, we get the tractability and convex approximation of the individual above chance constraint. The corresponding conditional value-at-value is defined as follows:

$$
\mathbb{P}-\operatorname{CVaR}_{\epsilon}(g(\mathbf{x}, \boldsymbol{\xi}))=\inf _{\beta \in \mathbb{R}}\left\{\beta+\frac{1}{\epsilon} \mathbb{E}_{\mathbb{P}}\left[(g(\mathbf{x}, \boldsymbol{\xi})-\beta)^{+}\right]\right\} .
$$

Next, we show that CVaR can be used to construct convex approximation for general chance constraint. By definition (7), we have

$$
\mathbb{P}\left(g(\mathbf{x}, \boldsymbol{\xi}) \leq \mathbb{P}-\mathrm{CVaR}_{\epsilon}(g(\mathbf{x}, \boldsymbol{\xi}))\right) \geq 1-\epsilon .
$$

Then

$$
\mathbb{P}-\mathrm{CVaR}_{\epsilon}(g(\mathbf{x}, \boldsymbol{\xi})) \leq 0 \Longrightarrow \mathbb{P}(g(\mathbf{x}, \boldsymbol{\xi}) \leq 0) \geq 1-\epsilon .
$$

Thus, from (9), we obtain

$$
\sup _{\mathbb{P} \in \mathscr{P}} \mathbb{P}-\mathrm{CVaR}_{\epsilon}(g(\mathbf{x}, \boldsymbol{\xi})) \leq 0 \Longrightarrow \inf _{\mathbb{P} \in \mathscr{P}} \mathbb{P}(g(\mathbf{x}, \boldsymbol{\xi}) \leq 0) \geq 1-\epsilon .
$$

Therefore, the worst-case constraint on the left hand side constitutes a conservative approximation for the distributionally robust chance constraint on the right hand side. The above discussion makes us define a feasible set as follows: $\Theta_{g}=\left\{\mathbf{x} \in \mathbb{R}^{n}: \sup _{\mathbb{P} \in \mathscr{P}} \mathbb{P}-\mathrm{CVaR}_{\epsilon}(g(\mathbf{x}, \boldsymbol{\xi})) \leq 0\right\}$.

Theorem 1. The feasible set $\Theta_{g}$ constitutes a conservative approximation for $\Pi_{g}$, in which $\Theta_{g} \subseteq \Pi_{g}$.

Lemma 2. For any fixed $x$, let $g(x, \xi): \mathbb{R}^{n} \times \mathbb{R}^{L} \rightarrow$ $\mathbb{R}$ be a measure function and F-integrable for all $F \in$ $\mathbb{D}\left(\mathbb{R}^{L}, \boldsymbol{\mu}_{0}, \boldsymbol{\Sigma}_{0}, \boldsymbol{\eta}\right)$. We define the worst-case expectation problem as follows:

$$
\Psi(\mathbf{x}, \boldsymbol{\eta})=\sup _{F \in \mathbb{D}} \int_{\mathbb{R}^{L}} \max (0, g(\mathbf{x}, \boldsymbol{\xi})) d F(\boldsymbol{\xi}) .
$$


Consequently, the problem can be rewritten into the following:

$$
\left.\begin{array}{ll}
\underset{y_{0}, \mathbf{y}_{1}, \mathbf{y}_{2}, \mathbf{Y}_{1}, \mathbf{Y}_{2}}{ } & \left\langle\Omega_{+},\left(\begin{array}{cc}
\mathbf{Y}_{1} & \frac{1}{2} \mathbf{y}_{1} \\
\frac{1}{2} \mathbf{y}_{1}^{T} & y_{0}
\end{array}\right)\right\rangle \\
& -\left\langle\Omega_{-},\left(\begin{array}{cc}
\mathbf{Y}_{2} & \frac{1}{2} \mathbf{y}_{2} \\
\frac{1}{2} \mathbf{y}_{2}^{T} & 0
\end{array}\right)\right\rangle
\end{array}\right\}
$$

where

$$
\begin{aligned}
& \Omega_{+}=\left(\begin{array}{cc}
\boldsymbol{\Sigma}_{0}+\left(\boldsymbol{\mu}_{0}+\boldsymbol{\eta}\right)\left(\boldsymbol{\mu}_{0}+\boldsymbol{\eta}\right)^{T} & \boldsymbol{\mu}_{0}+\boldsymbol{\eta} \\
\boldsymbol{\mu}_{0}^{T}+\boldsymbol{\eta}^{T} & 1
\end{array}\right), \\
& \Omega_{-}=\left(\begin{array}{cc}
\boldsymbol{\Sigma}_{0}+\left(\boldsymbol{\mu}_{0}-\boldsymbol{\eta}\right)\left(\boldsymbol{\mu}_{0}-\boldsymbol{\eta}\right)^{T} & \boldsymbol{\mu}_{0}-\boldsymbol{\eta} \\
\boldsymbol{\mu}_{0}^{T}-\boldsymbol{\eta}^{T} & 1
\end{array}\right) .
\end{aligned}
$$

Proof. The worst-case expectation $\Psi(\mathbf{x}, \boldsymbol{\eta})$ can equivalently be unfolded as

$$
\begin{array}{ll}
\sup _{F \in \mathscr{D}} & \int_{\mathbb{R}^{L}} \max (0, g(\mathbf{x}, \boldsymbol{\xi})) d F(\boldsymbol{\xi}) \\
\text { s.t. } & \int_{\mathbb{R}^{L}} d F(\boldsymbol{\xi})=1, \\
& \boldsymbol{\mu}_{0}-\boldsymbol{\eta} \leq \int_{\mathbb{R}^{L}} \boldsymbol{\xi} d F(\boldsymbol{\xi}) \leq \boldsymbol{\mu}_{0}+\boldsymbol{\eta}, \\
& \boldsymbol{\Sigma}_{0}+\left(\boldsymbol{\mu}_{0}-\boldsymbol{\eta}\right)\left(\boldsymbol{\mu}_{0}-\boldsymbol{\eta}\right)^{T} \\
& \preceq \int_{\mathbb{R}^{L}} \boldsymbol{\xi} \boldsymbol{\xi}^{T} d F(\boldsymbol{\xi}) \preceq \boldsymbol{\Sigma}_{0}+\left(\boldsymbol{\mu}_{0}+\boldsymbol{\eta}\right)\left(\boldsymbol{\mu}_{0}+\boldsymbol{\eta}\right)^{T} .
\end{array}
$$

We can formulate the Lagrangian dual problem of the above problem which takes the following form:

$$
\begin{aligned}
& \inf _{y_{0}, \mathbf{y}_{1}, \mathbf{y}_{2}, \mathbf{Y}_{1}, \mathbf{Y}_{2}} y_{0}+\mathbf{y}_{1}^{T}\left(\boldsymbol{\mu}_{0}+\boldsymbol{\eta}\right)-\mathbf{y}_{2}^{T}\left(\boldsymbol{\mu}_{0}-\boldsymbol{\eta}\right) \\
& +\left\langle\mathbf{Y}_{1}, \boldsymbol{\Sigma}_{\mathbf{0}}+\left(\boldsymbol{\mu}_{0}+\boldsymbol{\eta}\right)\left(\boldsymbol{\mu}_{0}+\boldsymbol{\eta}\right)^{T}\right\rangle \\
& -\left\langle\mathbf{Y}_{2}, \boldsymbol{\Sigma}_{\mathbf{0}}+\left(\boldsymbol{\mu}_{0}-\boldsymbol{\eta}\right)\left(\boldsymbol{\mu}_{0}-\boldsymbol{\eta}\right)^{T}\right\rangle \\
& \text { s.t. } \quad y_{0}+\left(\mathbf{y}_{1}-\mathbf{y}_{2}\right)^{T} \mathbf{y}_{1}+\left\langle\boldsymbol{\xi} \boldsymbol{\xi}^{T}, \mathbf{Y}_{1}-\mathbf{Y}_{2}\right\rangle \\
& \geq \max (0, g(x, \xi)), \quad \forall \xi \in \mathbb{R}^{L}, \\
& \mathbf{y}_{1} \geq 0, \quad \mathbf{y}_{2} \geq 0, \quad \mathbf{Y}_{1} \geq 0, \quad \mathbf{Y}_{2} \geq 0,
\end{aligned}
$$

where $y_{0} \in \mathbb{R}, \mathbf{y}_{1}, \mathbf{y}_{2} \in \mathbb{R}^{L}$, and $\mathbf{Y}_{1}, \mathbf{Y}_{2} \in \mathbb{S}^{L}$ are the dual variables for the constraints, respectively. It is obvious that the conditions on $\boldsymbol{\eta}$ and $\boldsymbol{\Sigma}_{0}$ are sufficient to ensure that the Dirac measure $\delta_{\boldsymbol{\mu}_{0}}$ lies in the relative interior of the feasible set of the above original problem. It implies that $\Psi(\mathbf{x}, \boldsymbol{\eta})$ must be equal to the optimal value of the above dual problem. Rewriting the above model into the form of LMIS, we have

$$
\begin{aligned}
& \inf _{y_{0}, \mathbf{y}_{1}, \mathbf{y}_{2}, \mathbf{Y}_{1}, \mathbf{Y}_{2}}\left\langle\Omega_{+},\left(\begin{array}{cc}
\mathbf{Y}_{1} & \frac{1}{2} \mathbf{y}_{1} \\
\frac{1}{2} \mathbf{y}_{1}^{T} & y_{0}
\end{array}\right)\right\rangle \\
& -\left\langle\Omega_{-},\left(\begin{array}{cc}
\mathbf{Y}_{2} & \frac{1}{2} \mathbf{y}_{2} \\
\frac{1}{2} \mathbf{y}_{2}^{T} & 0
\end{array}\right)\right\rangle \\
& \text { s.t. } \\
& \left(\begin{array}{c}
\boldsymbol{\xi} \\
1
\end{array}\right)^{T}\left(\left(\begin{array}{cc}
\mathbf{Y}_{1} & \frac{1}{2} \mathbf{y}_{1} \\
\frac{1}{2} \mathbf{y}_{1}^{T} & y_{0}
\end{array}\right)-\left(\begin{array}{cc}
\mathbf{Y}_{2} & \frac{1}{2} \mathbf{y}_{2} \\
\frac{1}{2} \mathbf{y}_{2}^{T} & 0
\end{array}\right)\right) \\
& \times\left(\begin{array}{l}
\boldsymbol{\xi} \\
1
\end{array}\right) \geq \max (g(\mathbf{x}, \boldsymbol{\xi}), 0), \quad \forall \boldsymbol{\xi} \in \mathbb{R}^{L}, \\
& \mathbf{y}_{1} \geq 0, \quad \mathbf{y}_{2} \geq 0, \quad \mathbf{Y}_{1} \geq 0, \quad \mathbf{Y}_{2} \geq 0 .
\end{aligned}
$$

Actually, we complete this proof when we divide the first inequality into two parts.

We define the feasible set $\Pi^{\text {JCC }}$ of the distributionally robust joint chance constraint as

$$
\begin{array}{r}
\Pi^{\mathrm{JCC}}=\left\{x \in \mathbb{R}^{n}: \inf _{\mathbb{P} \in \mathscr{P}} \mathbb{P}\left(w_{i}^{0}(\mathbf{x})+\mathbf{w}_{i}(\mathbf{x})^{T} \boldsymbol{\xi} \leq 0,\right.\right. \\
\forall i=1,2, \ldots, m) \geq 1-\epsilon\} .
\end{array}
$$

A popular approximation for $\Pi^{\mathrm{JCC}}$ is based on Bonferroni' inequality, but this method can be overly conservative even if 
$\epsilon$ is divided among the $m$ individual chance constraints, and Chen et al. [14] give an example which highlights this shortcoming. In order to mitigate the potential overconservatism of the Bonferroni approximation, we proposed the following approximation based on a combined inequality:

$$
\inf _{\mathbb{P} \in \mathscr{P}} \mathbb{P}\left(\max _{i=1,2, \ldots, m}\left\{w_{i}^{0}(\mathbf{x})+\mathbf{w}_{i}(\mathbf{x})^{T} \boldsymbol{\xi}\right\} \leq 0\right) \geq 1-\epsilon .
$$

The problem becomes an individual chance constraint which can be conservatively approximated by a Worst-Case CVaR constraint. We define the approximated set as follows:

$$
\begin{aligned}
\Theta^{\mathrm{JCC}}=\left\{\mathbf{x} \in \mathbb{R}^{n}\right. & : \sup _{\mathbb{P} \in \mathscr{P}} \mathbb{P}-\mathrm{CVaR}_{\epsilon} \\
& \left.\times\left(\max _{i=1,2, \ldots, m}\left\{w_{i}^{0}(\mathbf{x})+\mathbf{w}_{i}(\mathbf{x})^{T} \xi\right\} \leq 0\right)\right\},
\end{aligned}
$$

which is a tight approximation for $\Pi^{\mathrm{JCC}}$. The following theorem proves that the set $\Theta^{\text {JCC }}$ has a tractable reformation in terms of LMIS and therefore promises to get out a convex approximation for $\Pi^{\mathrm{JCC}}$.

Theorem 3. The feasible set $\Theta^{J C C}$ can be written as

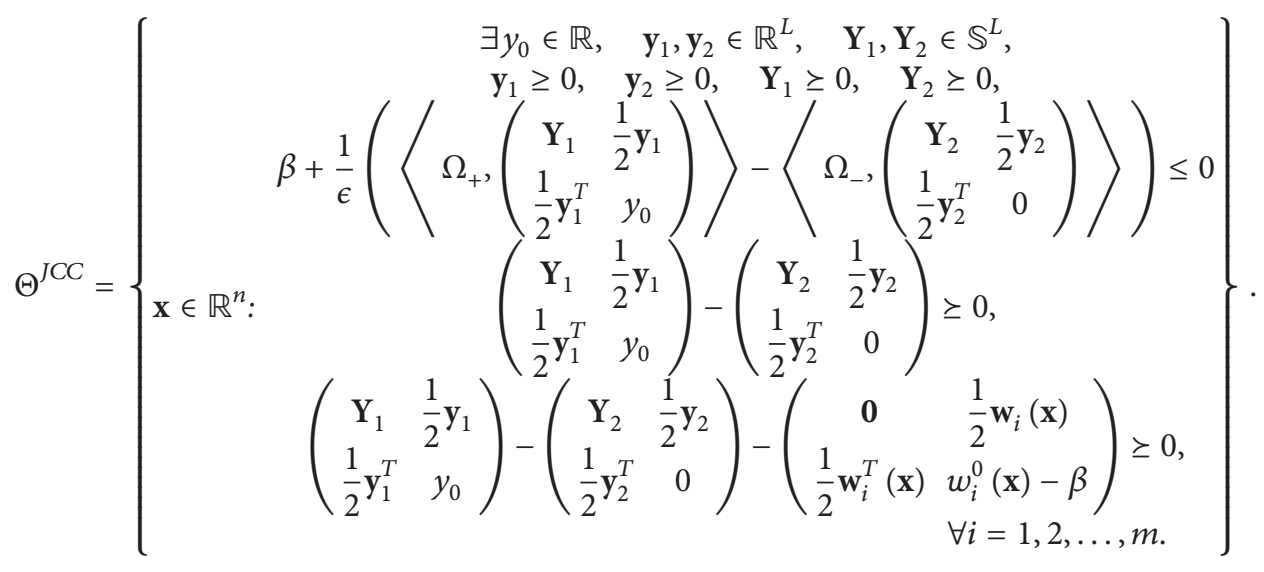

Proof. We find that the constraint $\mathbf{x} \in \Theta^{\mathrm{ICC}}$ is coinciding with $\Upsilon(\mathbf{x}) \leq 0$, where

$\Upsilon(\mathbf{x})$

$$
\begin{aligned}
& =\sup _{\mathbb{P} \in \mathscr{P}} \mathbb{P}-\mathrm{CVaR}_{\epsilon}\left(\max _{i=1,2, \ldots, m}\left\{w_{i}^{0}(\mathbf{x})+\mathbf{w}_{i}(\mathbf{x})^{T} \boldsymbol{\xi}\right\}\right) \\
& =\sup _{\mathbb{P} \in \mathscr{P}} \inf _{\beta \in \mathbb{R}}\left\{\beta+\frac{1}{\epsilon} \mathbb{E}_{\mathbb{P}}\left[\left(\max _{i=1,2, \ldots, m}\left\{w_{i}^{0}(\mathbf{x})+\mathbf{w}_{i}(\mathbf{x})^{T} \boldsymbol{\xi}\right\}-\beta\right)^{+}\right]\right\} .
\end{aligned}
$$

Actually, we know that $\beta+(1 / \epsilon) \mathbb{E}_{\mathbb{P}}\left[\left(\max _{i=1,2, \ldots, m}\left\{w_{i}^{0}(\mathbf{x})+\right.\right.\right.$ $\left.\left.\left.\mathbf{w}_{i}(\mathbf{x})^{T} \boldsymbol{\xi}\right\}-\beta\right)^{+}\right]$is real valued, convex in $\beta$ and linear in $\mathbb{P}$, where $\mathscr{P}$ is weakly compact [15]. Then, interchanging the $\sup _{\mathbb{P} \in \mathscr{P}}$ and $\inf _{\beta \in \mathbb{R}}$ operators leads to an equivalent formulation of the Worst-Case CVaR problem:

$$
\inf _{\beta \in \mathbb{R}}\left\{\beta+\frac{1}{\epsilon_{\mathbb{P} \in \mathscr{P}}} \sup _{\mathbb{P}}\left[\left(\max _{i=1,2, \ldots, m}\left\{w_{i}^{0}(\mathbf{x})+\mathbf{w}_{i}(\mathbf{x})^{T} \boldsymbol{\xi}\right\}-\beta\right)^{+}\right]\right\} .
$$

Firstly, we deal with the subordinate maximization problem in (22); we can derive an SDP reformulation for the worstcase expectation problem

$$
\sup _{\mathbb{P} \in \mathscr{P}} \mathbb{E}_{\mathbb{P}}\left[\left(\max _{i=1,2, \ldots, m}\left\{w_{i}^{0}(\mathbf{x})+\mathbf{w}_{i}(\mathbf{x})^{T} \boldsymbol{\xi}\right\}-\beta\right)^{+}\right]
$$

For any fixed $x$ and $\beta$, as shown in Lemma 2, the above subordinate maximization problem in (22) can be rewritten into

$$
\begin{aligned}
\underset{y_{0}, \mathbf{y}_{1}, \mathbf{y}_{2}, \mathbf{Y}_{1}, \mathbf{Y}_{2}}{\ln _{+}} & \left\langle\Omega_{+},\left(\begin{array}{cc}
\mathbf{Y}_{1} & \frac{1}{2} \mathbf{y}_{1} \\
\frac{1}{2} \mathbf{y}_{1}^{T} & y_{0}
\end{array}\right)\right\rangle \\
& -\left\langle\Omega_{-},\left(\begin{array}{cc}
\mathbf{Y}_{2} & \frac{1}{2} \mathbf{y}_{2} \\
\frac{1}{2} \mathbf{y}_{2}^{T} & 0
\end{array}\right)\right\rangle
\end{aligned}
$$


s.t.

$$
\begin{gathered}
\mathbf{y}_{1} \geq 0, \quad \mathbf{y}_{2} \geq 0, \quad \mathbf{Y}_{1} \geq 0, \quad \mathbf{Y}_{2} \geq 0 \\
\left(\begin{array}{cc}
\mathbf{Y}_{1} & \frac{1}{2} \mathbf{y}_{1} \\
\frac{1}{2} \mathbf{y}_{1}^{T} & y_{0}
\end{array}\right)-\left(\begin{array}{cc}
\mathbf{Y}_{2} & \frac{1}{2} \mathbf{y}_{2} \\
\frac{1}{2} \mathbf{y}_{2}^{T} & 0
\end{array}\right) \geq 0, \\
\left(\begin{array}{c}
\boldsymbol{\xi} \\
1
\end{array}\right)^{T}\left(\left(\begin{array}{cc}
\mathbf{Y}_{1} & \frac{1}{2} \mathbf{y}_{1} \\
\frac{1}{2} \mathbf{y}_{1}^{T} & y_{0}
\end{array}\right)-\left(\begin{array}{cc}
\mathbf{Y}_{2} & \frac{1}{2} \mathbf{y}_{2} \\
\frac{1}{2} \mathbf{y}_{2}^{T} & 0
\end{array}\right)\right)\left(\begin{array}{l}
\boldsymbol{\xi} \\
1
\end{array}\right) \\
\geq \max _{i=1,2, \ldots, m}\left\{w_{i}^{0}(\mathbf{x})+\mathbf{w}_{i}(\mathbf{x})^{T} \boldsymbol{\xi}\right\}-\beta, \\
\forall \boldsymbol{\xi} \in \mathbb{R}^{L} .
\end{gathered}
$$

The last inequality constraint in the above problem can be expanded into $m$ simpler inequality. Representing the subordinate worst-case expectation problem in (22), we get

$$
\begin{aligned}
\inf _{y_{0}, \mathbf{y}_{1}, \mathbf{y}_{2}, \mathbf{Y}_{1}, \mathbf{Y}_{2}} \beta+\frac{1}{\epsilon} & \left(\left\langle\Omega_{+},\left(\begin{array}{cc}
\mathbf{Y}_{1} & \frac{1}{2} \mathbf{y}_{1} \\
\frac{1}{2} \mathbf{y}_{1}^{T} & y_{0}
\end{array}\right)\right\rangle\right. \\
& \left.\left.-\left\langle\Omega_{-},\left(\begin{array}{cc}
\mathbf{Y}_{2} & \frac{1}{2} \mathbf{y}_{2} \\
\frac{1}{2} \mathbf{y}_{2}^{T} & 0
\end{array}\right)\right\rangle\right)\right)
\end{aligned}
$$

s.t. $\quad \mathbf{y}_{1} \geq 0, \quad \mathbf{y}_{2} \geq 0, \quad \mathbf{Y}_{1} \geq 0, \quad \mathbf{Y}_{2} \geq 0$,

$$
\begin{gathered}
\left(\begin{array}{cc}
\mathbf{Y}_{1} & \frac{1}{2} \mathbf{y}_{1} \\
\frac{1}{2} \mathbf{y}_{1}^{T} & y_{0}
\end{array}\right)-\left(\begin{array}{cc}
\mathbf{Y}_{2} & \frac{1}{2} \mathbf{y}_{2} \\
\frac{1}{2} \mathbf{y}_{2}^{T} & 0
\end{array}\right) \geq 0 \\
\left(\begin{array}{cc}
\mathbf{Y}_{1} & \frac{1}{2} \mathbf{y}_{1} \\
\frac{1}{2} \mathbf{y}_{1}^{T} & y_{0}
\end{array}\right)-\left(\begin{array}{cc}
\mathbf{Y}_{2} & \frac{1}{2} \mathbf{y}_{2} \\
\frac{1}{2} \mathbf{y}_{2}^{T} & 0
\end{array}\right) \\
-\left(\begin{array}{cc}
\mathbf{0} & \frac{1}{2} \mathbf{w}_{i}(\mathbf{x}) \\
\frac{1}{2} \mathbf{w}_{i}^{T}(\mathbf{x}) & w_{i}^{0}(\mathbf{x})-\beta
\end{array}\right) \geq 0 \\
\forall i=1,2, \ldots, m .
\end{gathered}
$$

Thus, we can easily get the exact representation of $\Theta^{\text {JCC }}$. This completes the proof.

Remark 4. When $m=1$, we set $\boldsymbol{\eta}=0$, which implies that we have the exact first- and second-order moments information for the individual chance constrained problem. We can get an exact approximation for $\Pi^{\mathrm{JCC}}$ :

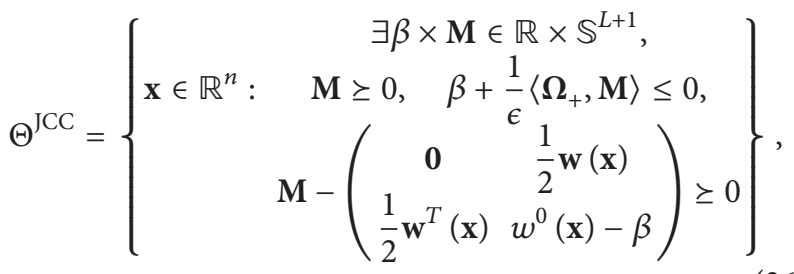

where we denote $\mathbf{M}=\left(\begin{array}{cc}\mathbf{Y}_{1}-\mathbf{Y}_{2} \\ (1 / 2)\left(\mathbf{y}_{1}-\mathbf{y}_{2}\right)^{T}\end{array} y_{0}^{(1 / 2)\left(\mathbf{y}_{1}-\mathbf{y}_{2}\right)}\right) \in \mathbb{S}^{L}$. This representation is exactly the one in [6]; then we can get the exact result of problem (5) (where $m=1$ ) by computing the above problem.

By now, we can compute the feasible set $\Theta^{\text {JCC }}$ by solving a tractable SDP. We appreciate that many modern methods can be used to solve such a convex problem to any precision $\epsilon$ in polynomial time and we can use YALMIP [16] to solve it in practice.

Consider the robust individual chance constraint (18) which represents the robust joint chance constraint (17). In convex programming, we use the max piecewise linear function to approximate a convex function frequently; there, we use a quadratic function to approximate the max function in the chance constraint (18) inversely. Note that $\mathfrak{h}(\xi)=$ $\boldsymbol{\xi}^{T} \mathbf{H} \boldsymbol{\xi}+\boldsymbol{\xi}^{T} \mathbf{h}+h^{0}$ that satisfies

(i) $\mathfrak{h}(\boldsymbol{\xi}) \geq \max _{i=1,2, \ldots, m}\left\{w_{i}^{0}(\mathbf{x})+\mathbf{w}_{i}(\mathbf{x})^{T} \boldsymbol{\xi}\right\}, \quad \forall \boldsymbol{\xi} \in \mathbb{R}^{L}$

$\Longleftrightarrow \mathfrak{h}(\boldsymbol{\xi}) \geq w_{i}^{0}(\mathbf{x})+\mathbf{w}_{i}(\mathbf{x})^{T} \boldsymbol{\xi}, \quad \forall \boldsymbol{\xi} \in \mathbb{R}^{L}, i=1,2, \ldots, m$,

(ii) $\inf _{\mathbb{P} \in \mathscr{P}} \mathbb{P}\left(\mathfrak{h}(\boldsymbol{\xi})=\boldsymbol{\xi}^{T} \mathbf{H} \boldsymbol{\xi}+\boldsymbol{\xi}^{T} \mathbf{h}+h^{0} \leq 0\right) \geq 1-\epsilon$.

For better argumentation, we define

$$
\begin{aligned}
& \Theta_{\mathrm{Q}}^{\text {JCC }} \\
& =\left\{\mathbf{x} \in \mathbb{R}^{n}: \exists \mathbf{H} \in \mathbb{S}^{L}, \mathbf{h} \in \mathbb{R}^{L}, h^{0} \in \mathbb{R},\right. \\
& \left.\quad \mathfrak{h}(\boldsymbol{\xi})=\boldsymbol{\xi}^{T} \mathbf{H} \boldsymbol{\xi}+\boldsymbol{\xi}^{T} \mathbf{h}+h^{0} \text { satisfies (27) and (28) }\right\} .
\end{aligned}
$$

Actually, it is easy to find that the feasible set $\Theta_{Q}^{\text {JCC }}$ constitutes a conservative approximation for $\Theta^{\text {JCC }}$, which means $\Theta_{Q}^{\text {JCC }} \subseteq \Theta^{\text {JCC }}$.

Theorem 5. The feasible set $\Theta_{\mathrm{Q}}^{I C C}$ can be written as 


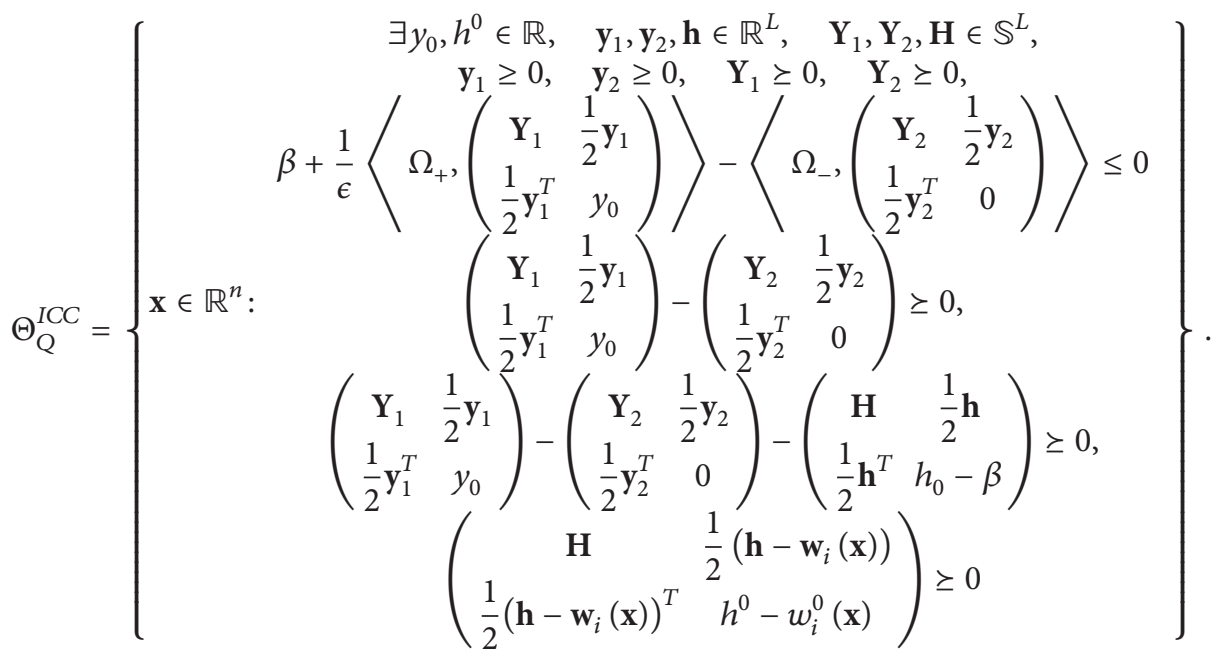

Moreover, we find $\Theta_{\mathrm{Q}}^{J C C}=\Theta^{I C C}$.

Proof. By a similar discussion as before, we get that the robust quadratic chance constraint (28) is equivalent to the WorstCase CVaR constraint:

$$
\begin{aligned}
& \sup _{\mathbb{P} \in \mathscr{P}} \mathbb{P}-\operatorname{CVaR}_{\epsilon}\left(\boldsymbol{\xi}^{T} \mathbf{H} \boldsymbol{\xi}+\boldsymbol{\xi}^{T} \mathbf{h}+h^{0}\right) \\
& =\inf _{\beta \in \mathbb{R}}\left\{\beta+\frac{1}{\epsilon_{\mathbb{P} \in \mathscr{P}}} \sup _{\mathbb{P}}\left[\left(\boldsymbol{\xi}^{T} \mathbf{H} \boldsymbol{\xi}+\boldsymbol{\xi}^{T} \mathbf{h}+h^{0}-\beta\right)^{+}\right]\right\} \leq 0 .
\end{aligned}
$$

We know that the above inequality can be reformulated as

$$
\begin{aligned}
& \sup _{\mathbb{P} \in \mathscr{P}} \mathbb{P}-\mathrm{CVaR}_{\epsilon}\left(\boldsymbol{\xi}^{T} \mathbf{H} \boldsymbol{\xi}+\boldsymbol{\xi}^{T} \mathbf{h}+h^{0}\right) \\
& =\inf _{y_{0}, \mathbf{y}_{1}, \mathbf{y}_{2}, \mathbf{Y}_{1}, \mathbf{Y}_{2}} \beta+\frac{1}{\epsilon}\left\langle\Omega_{+},\left(\begin{array}{cc}
\mathbf{Y}_{1} & \frac{1}{2} \mathbf{y}_{1} \\
\frac{1}{2} \mathbf{y}_{1}^{T} & y_{0}
\end{array}\right)\right\rangle \\
& -\left\langle\Omega_{-},\left(\begin{array}{cc}
\mathbf{Y}_{2} & \frac{1}{2} \mathbf{y}_{2} \\
\frac{1}{2} \mathbf{y}_{2}^{T} & 0
\end{array}\right)\right\rangle \\
& \text { s.t. } \quad \mathbf{y}_{1} \geq 0, \quad \mathbf{y}_{2} \geq 0, \quad \mathbf{Y}_{1} \geq 0, \quad \mathbf{Y}_{2} \geq 0 \text {, } \\
& \left(\begin{array}{cc}
\mathbf{Y}_{1} & \frac{1}{2} \mathbf{y}_{1} \\
\frac{1}{2} \mathbf{y}_{1}^{T} & y_{0}
\end{array}\right)-\left(\begin{array}{cc}
\mathbf{Y}_{2} & \frac{1}{2} \mathbf{y}_{2} \\
\frac{1}{2} \mathbf{y}_{2}^{T} & 0
\end{array}\right) \geq 0, \\
& \left(\begin{array}{cc}
\mathbf{Y}_{1} & \frac{1}{2} \mathbf{y}_{1} \\
\frac{1}{2} \mathbf{y}_{1}^{T} & y_{0}
\end{array}\right)-\left(\begin{array}{cc}
\mathbf{Y}_{2} & \frac{1}{2} \mathbf{y}_{2} \\
\frac{1}{2} \mathbf{y}_{2}^{T} & 0
\end{array}\right) \\
& -\left(\begin{array}{cc}
\mathbf{H} & \frac{1}{2} \mathbf{h} \\
\frac{1}{2} \mathbf{h}^{T} & h_{0}-\beta
\end{array}\right) \succeq 0 .
\end{aligned}
$$

Note that the constraints in (27) are equivalent to

$$
\left(\begin{array}{cc}
\mathbf{H} & \frac{1}{2}\left(\mathbf{h}-\mathbf{w}_{i}(\mathbf{x})\right) \\
\frac{1}{2}\left(\mathbf{h}-\mathbf{w}_{i}(\mathbf{x})\right)^{T} & h^{0}-w_{i}^{0}(\mathbf{x})
\end{array}\right) \geq 0,
$$

Thus, we can get the tractable form of $\Theta_{Q}^{\text {JCC }}$ in Theorem 5. The LMIs in Theorem 5 can be represented as

$$
\begin{gathered}
\left(\begin{array}{cc}
\mathbf{Y}_{1} & \frac{1}{2} \mathbf{y}_{1} \\
\frac{1}{2} \mathbf{y}_{1}^{T} & y_{0}
\end{array}\right)-\left(\begin{array}{cc}
\mathbf{Y}_{2} & \frac{1}{2} \mathbf{y}_{2} \\
\frac{1}{2} \mathbf{y}_{2}^{T} & 0
\end{array}\right)+\left(\begin{array}{cc}
\mathbf{0} & \mathbf{0} \\
\mathbf{0} & \beta
\end{array}\right) \\
\succeq\left(\begin{array}{cc}
\mathbf{H} & \mathbf{h} \\
\mathbf{h}^{T} & h^{0}
\end{array}\right) \succeq\left(\begin{array}{cc}
\mathbf{0} & \frac{1}{2} \mathbf{w}_{i}(\mathbf{x}) \\
\frac{1}{2} \mathbf{w}_{i}(\mathbf{x})^{T} & w_{i}^{0}(\mathbf{x})
\end{array}\right) .
\end{gathered}
$$

Finally, we get $\Theta_{Q}^{\text {JCC }}=\Theta^{\text {ICC }}$ with vanishing the middle matrix which is formed by the components of $\mathfrak{h}(\xi)$.

This theorem presents that the approximation of a distributionally robust joint chance constraint by a Worst-Case CVaR constraint is equivalent to the approximation of the max function implied by the joint chance constraint by a quadratic majorant.

Let us consider the case that $\xi$ have finite support information which can be present as the matrix $\zeta=$ $\left(\boldsymbol{\xi}_{1}, \boldsymbol{\xi}_{2}, \ldots, \boldsymbol{\xi}_{N}\right)$. Let the measures $\underline{\mathbf{p}}=\left(\underline{p}_{1}, \ldots, \underline{p}_{N}\right)$ and $\overline{\mathbf{p}}=$ $\left(\bar{p}_{1}, \ldots, \bar{p}_{N}\right)$ be two known vectors which give the bounds of the probability measure with $\underline{\mathbf{p}}<\overline{\mathbf{p}}$. The ambiguity distributional set can be defined as

$$
\begin{aligned}
& \mathbb{D}_{1}\left(\mathbb{R}^{L}, \boldsymbol{\mu}_{0}, \boldsymbol{\eta}\right) \\
& =\left\{\mathbf{p}: \mathbf{p}^{T} \mathbf{e}=1, \underline{\mathbf{p}} \leq \mathbf{p} \leq \overline{\mathbf{p}}, \boldsymbol{\mu}_{0}-\boldsymbol{\eta} \leq \boldsymbol{\zeta} \mathbf{p} \leq \boldsymbol{\mu}_{0}+\boldsymbol{\eta}\right\} .
\end{aligned}
$$


Consider the minimax problem (22) with the above ambiguity distributional set; we have the following results.
Theorem 6. $\Theta^{J C C}$ has the following tractable reformulation in terms of LMIs:

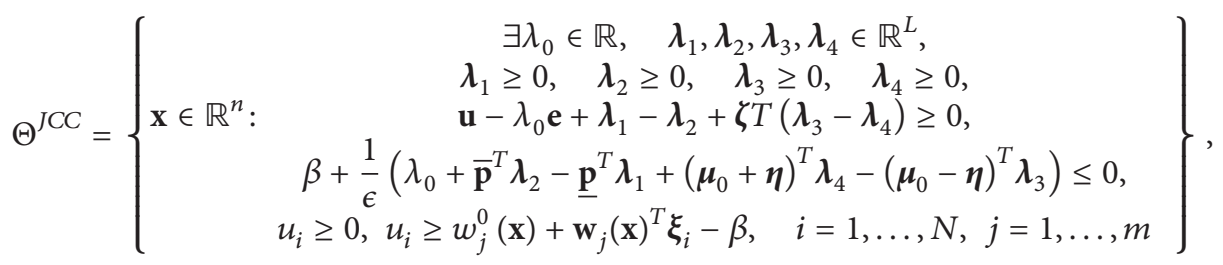

where $\mathbf{e}$ denotes the vector of ones.

Proof. Consider the subordinate maximization problem in (22); we can derive a reformulation for the worst-case expectation problem:

$$
\begin{aligned}
& \sup _{\mathbb{P} \in \mathbb{D}_{1}} \mathbb{E}_{\mathbb{P}}\left[\left(\max _{i=1,2, \ldots, m}\left\{w_{i}^{0}(\mathbf{x})+\mathbf{w}_{i}(\mathbf{x})^{T} \boldsymbol{\xi}\right\}-\beta\right)^{+}\right] \\
& =\sup _{\mathbb{P} \in \mathbb{D}_{1}} \sum_{i=1}^{N} p_{i}\left(\max _{i=1,2, \ldots, m}\left\{w_{i}^{0}(\mathbf{x})+\mathbf{w}_{i}(\mathbf{x})^{T} \boldsymbol{\xi}_{i}\right\}-\beta\right)^{+} .
\end{aligned}
$$

For any fixed $x$ and $\beta$, we can formulate the Lagrangian dual problem of the above linear programming. Actually, the standard duality theory guarantees that there is no duality gap between the above problem and its dual problem as

$$
\begin{array}{lr}
\min _{\lambda_{0}, \lambda_{1}, \lambda_{2}, \lambda_{3}, \lambda_{4}} & \beta+\frac{1}{\epsilon}\left(\lambda_{0}+\overline{\mathbf{p}}^{T} \boldsymbol{\lambda}_{2}-\underline{\mathbf{p}}^{T} \boldsymbol{\lambda}_{1}\right. \\
& \left.+\left(\boldsymbol{\mu}_{0}+\boldsymbol{\eta}\right)^{T} \boldsymbol{\lambda}_{4}-\left(\boldsymbol{\mu}_{0}-\boldsymbol{\eta}\right)^{T} \boldsymbol{\lambda}_{3}\right) \\
\text { s.t. } & \mathbf{u}-\lambda_{0} \mathbf{e}+\boldsymbol{\lambda}_{1}-\boldsymbol{\lambda}_{2}+\boldsymbol{\zeta} T\left(\boldsymbol{\lambda}_{3}-\boldsymbol{\lambda}_{4}\right) \geq 0, \\
& u_{i} \geq 0, \quad u_{i} \geq w_{j}^{0}(\mathbf{x})+\mathbf{w}_{j}(\mathbf{x})^{T} \xi_{i}-\beta, \\
& i=1, \ldots, N, \quad j=1, \ldots, m .
\end{array}
$$

\section{Conflict of Interests}

The author declares that there is no conflict of interests regarding the publication of this paper.

\section{Acknowledgments}

The author would like to thank Professor Nan-jing Huang for handling the review of this paper and two referees for helpful suggestions and comments. This work was supported by the Fundamental Research Funds for the Central Universities (2014NZYQN49).

\section{References}

[1] A. Charnes, W. W. Cooper, and G. H. Symonds, "Cost horizons and certainty equivalents: an approach to stochastic progrmming of heating oil," Managements Science, vol. 4, no. 3, pp. 235263, 1958.

[2] L. B. Miller and H. Wagnet, "Chance-constrained programming with joint constraints," Operations Research, vol. 13, no. 6, pp. 930-945, 1965.

[3] A. Prékopa, "On probabilistic constrained programming," in Proceedings of the Princeton Symposium on Mathematical Programming, pp. 113-138, Princeton University Press, Princeton, NJ, USA, 1970.

[4] F. Alizadeh and D. Goldfarb, "Second-order cone programming," Mathematical Programming Series B, vol. 95, no. 1, pp. 3-51, 2003.

[5] G. C. Calafiore and L. El Ghaoui, "On distributionally robust chance-constrained linear programs," Journal of Optimization Theory and Applications, vol. 130, no. 1, pp. 1-22, 2006.

[6] S. Zymler, D. Kuhn, and B. Rustem, "Distributionally robust joint chance constraints with second-order moment information," Mathematical Programming Series A, vol. 137, no. 1-2, pp. 167-198, 2013.

[7] A. Nemirovski and A. Shapiro, "Convex approximations of chance constrained programs," SIAM Journal on Optimization, vol. 17, no. 4, pp. 969-996, 2006.

[8] E. Erdoğan and G. Iyengar, "Ambiguous chance constrained problems and robust optimization," Mathematical Programming Series B, vol. 107, no. 1-2, pp. 37-61, 2006.

[9] A. Ben-Tal, L. El Ghaoui, and A. Nemirovski, Robust Optimization, Princeton University Press, Princeton, NJ, USA, 2009.

[10] D. Bertsimas, D. B. Brown, and C. Caramanis, "Theory and applications of robust optimization," SIAM Review, vol. 53, no. 3, pp. 464-501, 2011.

[11] A. Shapiro, D. Dentcheva, and A. Ruszczyński, Lecture on Stochastic Programming Modeling and Theory, Society for Industrial and Applied Mathematics, Philadelphia, Pa, USA, 2009.

[12] R. T. Rockafellar and S. Uryasev, "Conditional value-at-risk for general loss distributions," Journal of Banking and Finance, vol. 26, no. 7, pp. 1443-1471, 2002.

[13] H. Föllmer and A. Schied, Stochastic Finance: An Introduction in Discrete Time, Walter de Gruyter, Berlin, Germany, 2004.

[14] W. Chen, M. Sim, J. Sun, and C.-P. Teo, "From CVaR to uncertainty set: implications in joint chance-constrained optimization," Operations Research, vol. 58, no. 2, pp. 470-485, 2010.

[15] A. Shapiro, M. A. Goberna, and M. A. López, "On duality theory of conic linear problems," in Semi-Infinite Programming: Recent 
Advances, vol. 57, pp. 135-165, Kluwer Academic Publishers, Dordrecht, The Netherlands, 2001.

[16] J. Löfberg, "YALMIP: a toolbox for modeling and optimization in MATLAB," in Proceedings of the IEEE International Symposium on Computer Aided Control Systems Design (CACSD '04), Taipei, Taiwan, 2004. 


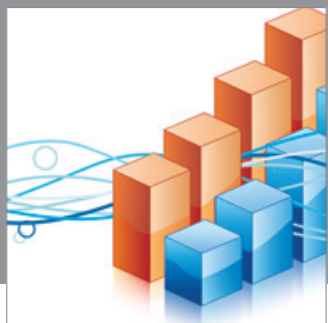

Advances in

Operations Research

mansans

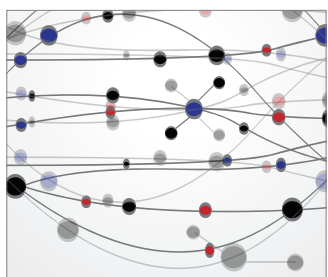

The Scientific World Journal
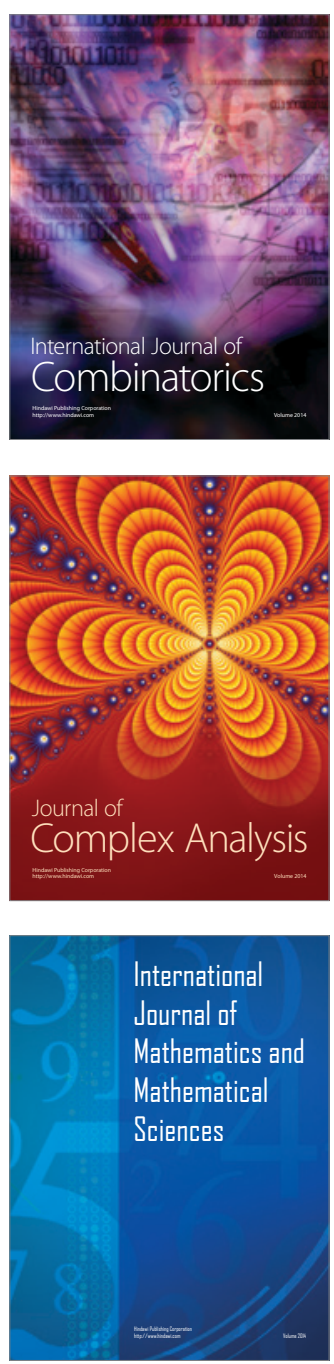
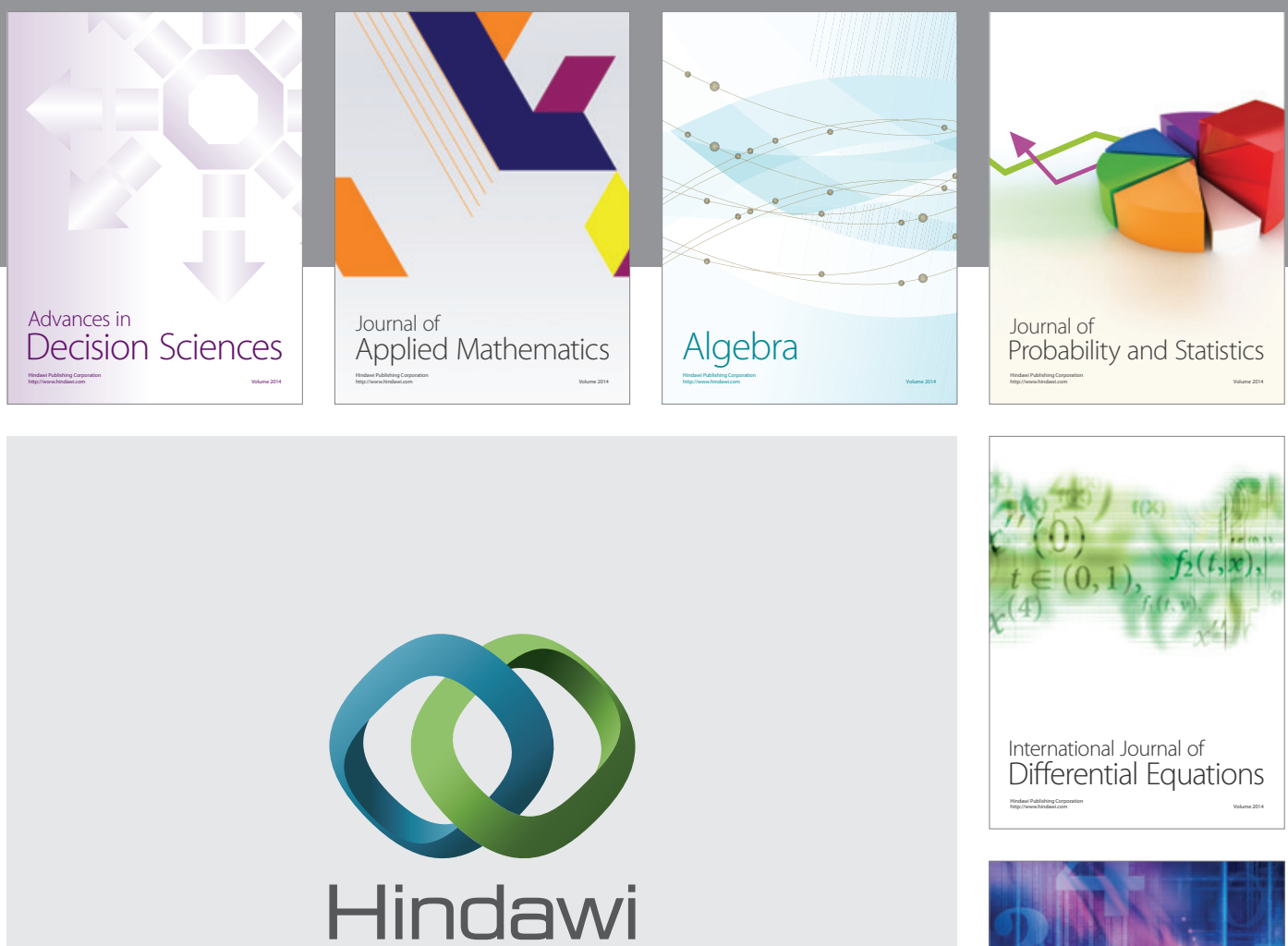

Submit your manuscripts at http://www.hindawi.com
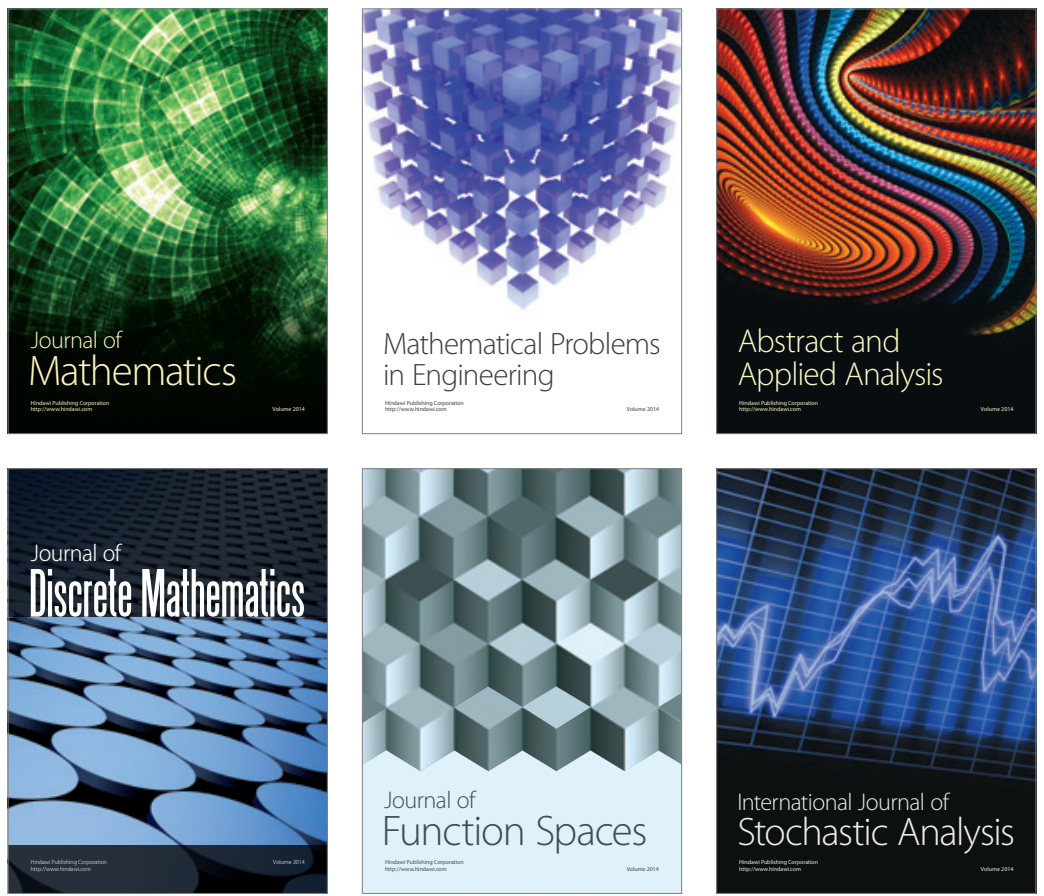

Journal of

Function Spaces

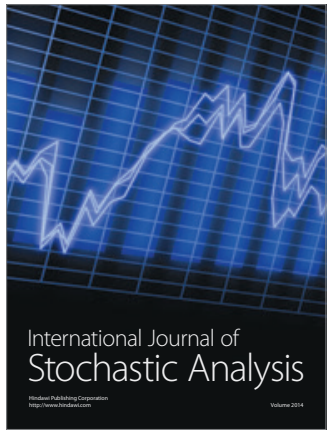

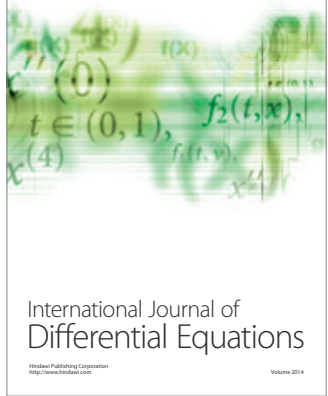
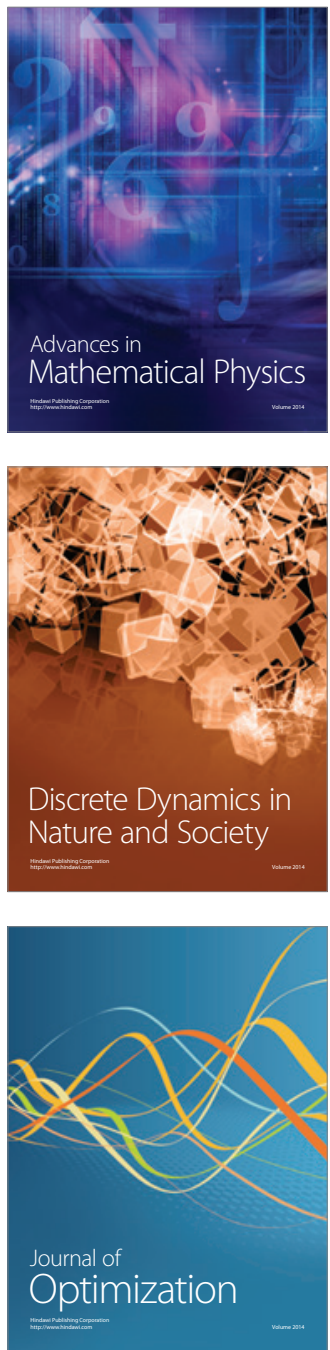